\title{
Sub-Phenotypes of Acute Kidney Injury: Do We Have Progress for Personalizing Care?
}

\author{
Matthew R. Thau ${ }^{a}$ Pavan K. Bhatraju ${ }^{a, b}$ \\ aDivision of Pulmonary, Critical Care and Sleep Medicine, Department of Medicine, University of Washington,

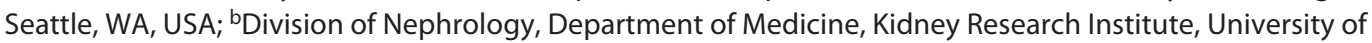 \\ Washington, Seattle, WA, USA
}

\section{Keywords}

Acute renal injury · Angiogenesis · Biomarkers .

Sub-phenotypes

\begin{abstract}
Acute kidney injury (AKI) is the most common form of organ dysfunction occurring in patients admitted to the intensive care unit and contributes significantly to poor long-term outcomes. Despite this public health impact, no effective pharmacotherapy exists for AKI. One reason may be that heterogeneity is present within AKI as currently defined, thereby concealing unique pathophysiologic processes specific to certain AKI populations. Supporting this notion, we and others have shown that diversity within the AKI clinical syndrome exists, and the "one-size-fits-all" approach by current diagnostic guidelines may not be ideal. A "precision medicine" approach that exploits an individual's genetic, biologic, and clinical characteristics to identify AKI sub-phenotypes may overcome such limitations. Identification of AKI subphenotypes may address a critical unmet clinical need in AKI by (1) improving risk prognostication, (2) identifying novel pathophysiology, and (3) informing a patient's likelihood of responding to current therapeutics or establishing new therapeutic targets to prevent and treat AKI. This review discusses the current state of phenotyping AKI and future directions.

(c) 2020 S. Karger AG, Basel
\end{abstract}

\section{Introduction}

Acute kidney injury (AKI) is common in hospitalized populations and associated with morbidity and mortality. Multiple observational studies have shown a link between AKI and the development of CKD, ESRD, cardiovascular disease, and mortality, even in subjects with reported renal recovery [1]. Numerous clinical trials have been conducted in an effort to identify disease-modifying therapies, yet no safe and effective pharmacotherapeutics have been identified. One reason for the lack of therapies may be that lumping patients with different AKI risk factors and pathophysiology may hide a treatment signal specific to certain biologically distinct AKI populations.

The Kidney Disease Improving Global Outcomes (KDIGO) consensus group defines AKI as an increase in serum $\mathrm{Cr}$ ( $\mathrm{SCr}$ ) of $\geq 0.3 \mathrm{mg} / \mathrm{dL}$ or $>50 \%$ of the baseline within a 48 -h period or a 7 -day time period. Although this definition has resulted in improved recognition and the discovery of critical concepts applicable to populations of

This work was performed at the University of Washington, Seattle, WA. Contribution from the AKI and CRRT 2020 Symposium at the 25th International Conference on Advances in Critical Care Nephrology, Manchester Grand Hyatt, San Diego, CA, USA, February 24-27, 2020. This symposium was supported in part by the NIDDK-funded University of Alabama at Birmingham-University of California San Diego O'Brien Center for Acute Kidney Injury Research (P30DK079337).

$\begin{aligned} & \text { karger@karger.com } \\ & \text { www.karger.com/nef }\end{aligned}$
Karger ${ }^{\prime /}$




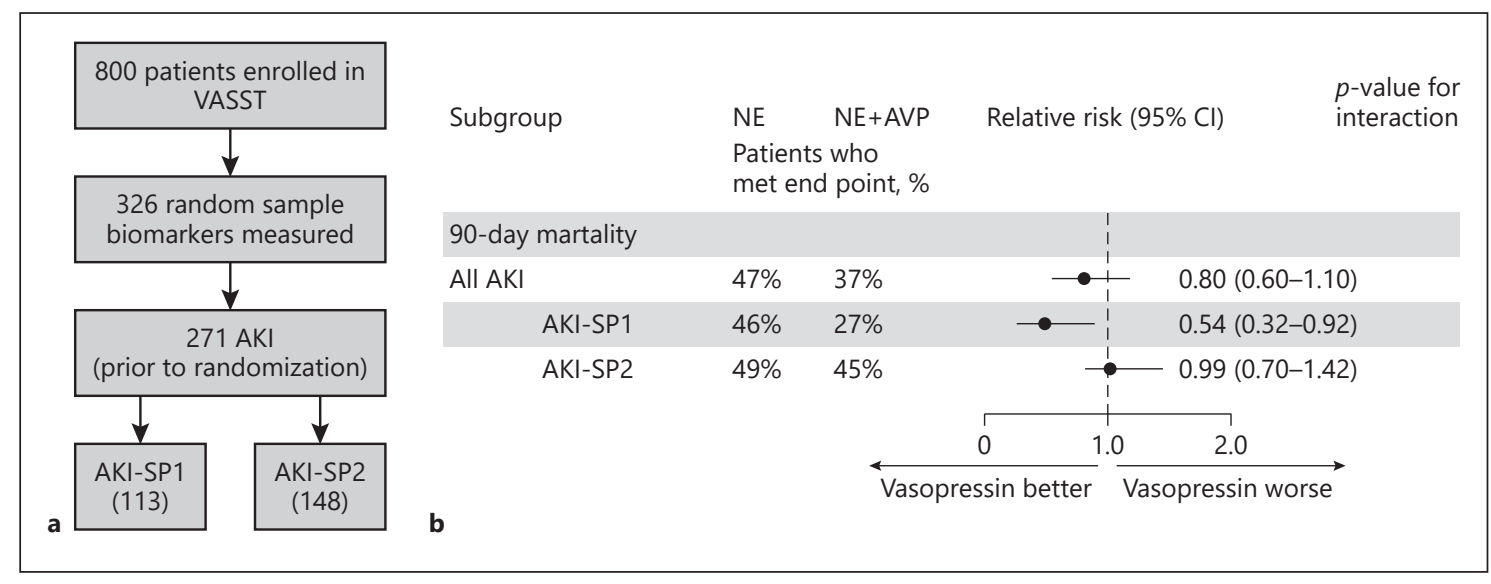

Fig. 1. Treatment interaction between AKI sub-phenotypes and vasopressor therapy in patients with septic shock. a Patients included in the secondary analysis of the VASST clinical trial. b Forest plot of the risk of 90-day mortality. Relative risk estimates were adjusted for APACHE II scores and norepinephrine dose prior to randomization. AKI, acute kidney injury; VASST, Vasopressin versus Norepinephrine in Septic Shock Study.

patients, it fails to stratify subjects with different underlying biology, recovery patterns, and subsequent outcomes. Identifying meaningful groups within the diverse syndrome of AKI may provide a deeper understanding of disease pathophysiology and aid in discovery of potential therapeutic targets.

\section{Clinical AKI Sub-Phenotypes}

Despite the broad definition for AKI, clinicians have intuitively "phenotyped" AKI into groups for years: prerenal AKI (i.e., "volume-responsive") and intrinsic AKI (i.e., acute tubular necrosis). Although conceptually straightforward, there is difficulty in reliably identifying these groups. A recent intervention, known as the "furosemide stress test," seeks to help clinicians identify these 2 AKI subgroups. The furosemide stress test challenges renal tubular function by administering high-dose furosemide and assessing the subsequent urine output response within $2 \mathrm{~h}$ of administration, thereby providing an indirect measure of functional nephrons [2]. In addition, a series of preclinical and clinical experiments attempted to demonstrate whether prerenal and acute tubular necrosis subgroups share similar pathogenesis or are distinct entities [3]. By developing murine models for prerenal and intrinsic AKI and performing complete RNA sequencing of mouse kidney tissue, $\mathrm{Xu}$ and colleagues [3] identified thousands of genes that were differentially regulated. The investigators then measured 40 corresponding candidate urinary proteins in clinical AKI and found different urinary protein concentrations between patients with volume-responsive and intrinsic AKI, suggesting that these 2 entities were distinct groups with differing pathophysiology.

Several research groups have sought to identify AKI subgroups based on kidney functional recovery after injury $[4,5]$. In 2016, Bhatraju and colleagues [4] identified 2 AKI sub-phenotypes based on the trajectory of SCr and "resolving" and "nonresolving" AKI. Patients with nonresolving AKI had higher hospital mortality, and in a subsequent analysis, patients with nonresolving AKI had poorer long-term outcomes, such as development and progression of $\mathrm{CKD}$, dialysis, and death, than patients with resolving AKI [6]. These associations were independent of the traditional KDIGO severity stage of AKI.

\section{Biomarker-Based AKI Sub-Phenotypes}

A number of blood and urinary biomarkers have been shown to predict the development of AKI, such as kidney injury molecule 1, neutrophil gelatinase-associated lipocalin, tissue inhibitor of metalloproteinase-2, and insulinlike growth factor-binding protein 7, and others [7]. More recently, studies have used these biomarkers to describe an emerging AKI phenotype called subclinical AKI, in which SCr does not rise, but urinary biomarkers are elevated [8]. Alternative approaches to identifying AKI subphenotypes include unsupervised clustering analyses of high-dimensional biological data, incorporating multiple biomarkers. In 2018, Bhatraju and colleagues [9] applied latent class analysis methodology to identify 2 novel AKI 
sub-phenotypes, AKI-SP1 and AKI-SP2, with the latter associated with a greater risk of 7-day renal nonrecovery, need for renal replacement therapy, and 28-day mortality than AKI-SP1. The investigators then developed a threebiomarker prediction model (ratio of angiopoietin-2/angiopoietin-1 and tumor necrosis factor receptor 1) to identify AKI sub-phenotypes. Next, the investigators used blood collected prior to randomization to identify AKI sub-phenotypes in the Vasopressin versus Norepinephrine in Septic Shock Study (VASST) clinical trial. Patients with AKI-SP1 had improved mortality with the early addition of vasopressin as opposed to norepinephrine only ( 27 vs. $46 \%, p=0.02$ ), but no benefit was observed for patients with AKI-SP2 (45 vs. $49 \%, p=0.99$ ), and the $p$ value for interaction was 0.05 (Fig. 1). These findings are notable, as the initial VASST study demonstrated no significant difference in the 28- or 90-day mortality rate between vasopressor therapy. Subsequently, Wiersema and colleagues [10] applied latent class analysis to the Finnish Acute Kidney Injury Cohort and identified similar AKI sub-phenotypes.

Another emerging tool to identify AKI sub-phenotypes includes machine-learning techniques using electronic medical record (EMR) data. Chaudhary and colleagues [11] used deep learning algorithms to extract data from the EMR and then used K-means clustering to identify 3 sepsis-associated AKI sub-phenotypes. In 4,001 patients admitted to the intensive care unit, they identified 3 AKI sub-phenotypes using routinely measured laboratory measurements and vital signs. These AKI sub-phe- notypes differentiated the risk of dialysis and 28-day mortality [11].

\section{Conclusion}

Substantial progress has been made in the identification of AKI sub-phenotypes. Different lines of evidence suggest that AKI is not one disease but a collection of many different sub-phenotypes with differing risk factors, underlying pathophysiology, and clinical outcomes. While some basic treatment strategies for AKI have improved outcomes, such as prevention of nephrotoxic agents, an improved classification of AKI using biologically relevant sub-phenotypes could lead to enhanced prognostication and predictive enrichment of clinical trials to identify much needed therapeutics for both the prevention and treatment of AKI.

\section{Conflict of Interest Statement}

The authors have no conflicts of interest.

\section{Funding Sources}

P.K.B. was supported by grants from the Digestive and Kidney Diseases K23DK116967 and an unrestricted gift to the Kidney Research Institute from the Northwest Kidney Centers.

\section{References}

1 Coca SG, Singanamala S, Parikh CR. Chronic kidney disease after acute kidney injury: a systematic review and meta-analysis. Kidney Int. 2012;81(5):442-8.

2 Koyner JL, Davison DL, Brasha-Mitchell E, Chalikonda DM, Arthur JM, Shaw AD, et al. Furosemide stress test and biomarkers for the prediction of AKI severity. J Am Soc Nephrol. 2015;26(8):2023-31.

$3 \mathrm{Xu} \mathrm{K}$, Rosenstiel P, Paragas N, Hinze C, Gao $\mathrm{X}$, Huai Shen $\mathrm{T}$, et al. Unique transcriptional programs identify subtypes of AKI. J Am Soc Nephrol. 2016;28(6):1729.

4 Bhatraju PK, Mukherjee P, Robinson-Cohen C, O’Keefe GE, Frank AJ, Christie JD, et al. Acute kidney injury subphenotypes based on creatinine trajectory identifies patients at increased risk of death. Crit Care. 2016;20(1): 372 .
5 Kellum JA, Sileanu FE, Bihorac A, Hoste EA, Chawla LS. Recovery after acute kidney injury. Am J Respir Crit Care Med. 2016;195(6): 784.

6 Bhatraju PK, Zelnick LR, Chinchilli VM, Moledina DG, Coca SG, Parikh CR, et al. Association between early recovery of kidney function after acute kidney injury and longterm clinical outcomes. JAMA Netw Open. 2020;3(4):e202682.

7 Malhotra R, Siew ED. Biomarkers for the early detection and prognosis of acute kidney injury. Clin J Am Soc Nephrol. 2017;12(1):14973.

8 Moledina DG, Parikh CR. Acute kidney injury following contrast administration in pediatric congenital heart disease patients: time to move beyond the serum creatinine. Catheter Cardiovasc Interv. 2018;84(4):620-11.
9 Bhatraju PK, Zelnick LR, Herting J, Katz R, Mikacenic C, Kosamo S, et al. Identification of acute kidney injury sub-phenotypes with differing molecular signatures and response to vasopressin therapy. Am J Respir Crit Care Med. 2019;199(7):863-72.

10 Wiersema R, Jukarainen S, Vaara ST, Poukkanen M, Lakkisto P, Wong $\mathrm{H}$, et al. Two subphenotypes of septic acute kidney injury are associated with different 90-day mortality and renal recovery. Crit Care. 2020;24(1):150.

11 Chaudhary K, Vaid A, Duffy A, Poojary P, Saha A, Chauhan K, et al. Utilization of deep learning for subphenotype identification in sepsis-associated acute kidney injury. Clin J Am Soc Nephrol. 